\title{
Quantifying Player-Induced Intonation Errors of the Steel String Acoustic Guitar
}

\author{
R.M. MOTTOLA ${ }^{1}$
}

\begin{abstract}
Data from a previously published study on same-note pitch variability of the steel string acoustic guitar were subjected to additional analysis to isolate and quantify the sources of intonation error. Pitch error components associated with the construction of the guitar, the player's tendency to play sharp, and the player's tendency to pitch variability were identified and isolated by simple arithmetic operations. The player-associated error components were found to be substantially related to the fret being played. Per-player sharping tendency values for the $G$ string ranged from $0-4$ cents on fret \#1, $0-6$ cents on fret \#5, and $0-10$ cents on fret \#12. Per player pitch variability mean values ranged from 0 - 2 cents on fret \#1, 1 - 4 cents on fret \#5, and 1 - 7 cents on fret \#12. Quantifying playerassociated pitch error tendencies may be useful in optimizing an instrument's intonation for that player.
\end{abstract}

\section{INTRODUCTION}

A previous paper[1] documented considerable pitch variability during sequential fretting of the same string and fret of a steel string acoustic guitar[1]. This article documents an analysis of the data set from [1] to determine and quantify the effects of this variability on modeled intonation.

Considerable research has been done with the aim of improving guitar intonation[2-8]. Much of this appears in guitar construction literature. These efforts generally propose mathematical models of guitar intonation and suggest compensatory modifications to the instrument based on these models. Modifications consist of changes in placement of the nut and bridge saddle, and/or changes in fret placement. With the exception of [8] none of the articles describe validation of their proposed models, either by physical pitch measurement or by evaluation of human pitch perception. In [8] the proposed model is validated by measuring pitches from a test fixture built to the proposed model. But the test fixture is a sonometer modified to include a guitar fretboard and it is not clear the extent to which fingering of this fixture is analogous to that of an actual guitar. As such, player-induced intonation error may be different for this fixture than for an actual steel string guitar.

Preliminary work by the author to validate models proposed in the literature on the steel string acoustic guitar resulted in two observations. The first is that there is considerable pitch variation among notes played at the same string/fret position, and the second is that the pitches of all notes are sharper than the nominal pitch of the string/fret position. These observations led to the experiment described in [1] which documented the extent of pitch variability on frets 1,5 and 12 of the G3 string of a steel string guitar with repeated fretting by 10 player subjects. That study did not attempt to document the initial observation that all fretted notes are sharper than their nominal pitches. The reason for this lack of documentation is that direct pitch measurement of a fretted note requires fretting, which is observed to result in pitch variation. Thus the process of measuring fretted note pitch affects the measurement.

In [1], summary statistics were calculated and the results were plotted. This study assesses those results and identifies pitch error components and proposes practical compensation techniques which may be added to existing intonation models to compensate for the noted variability.

\section{ANALYSIS}

Figure 1 is taken from and shows the results obtained in [1]. Note that in the figure, note error data is normalized by subtracting the population mean value from each error datum. This has the effect of offsetting the population mean values to zero error. This data normalization step aids in visual comparison of data for the three notes. It also aids in comparison of the data collected in that study with that found in the referenced articles on compensation. It should not be taken to imply that the actual pitch errors are centered about zero error.

\footnotetext{
${ }^{1}$ R.M. Mottola - Liutaio Mottola Stringed Instrument Design, Newton MA 02460 USA, rmm at LiutaioMottola dot com
} 


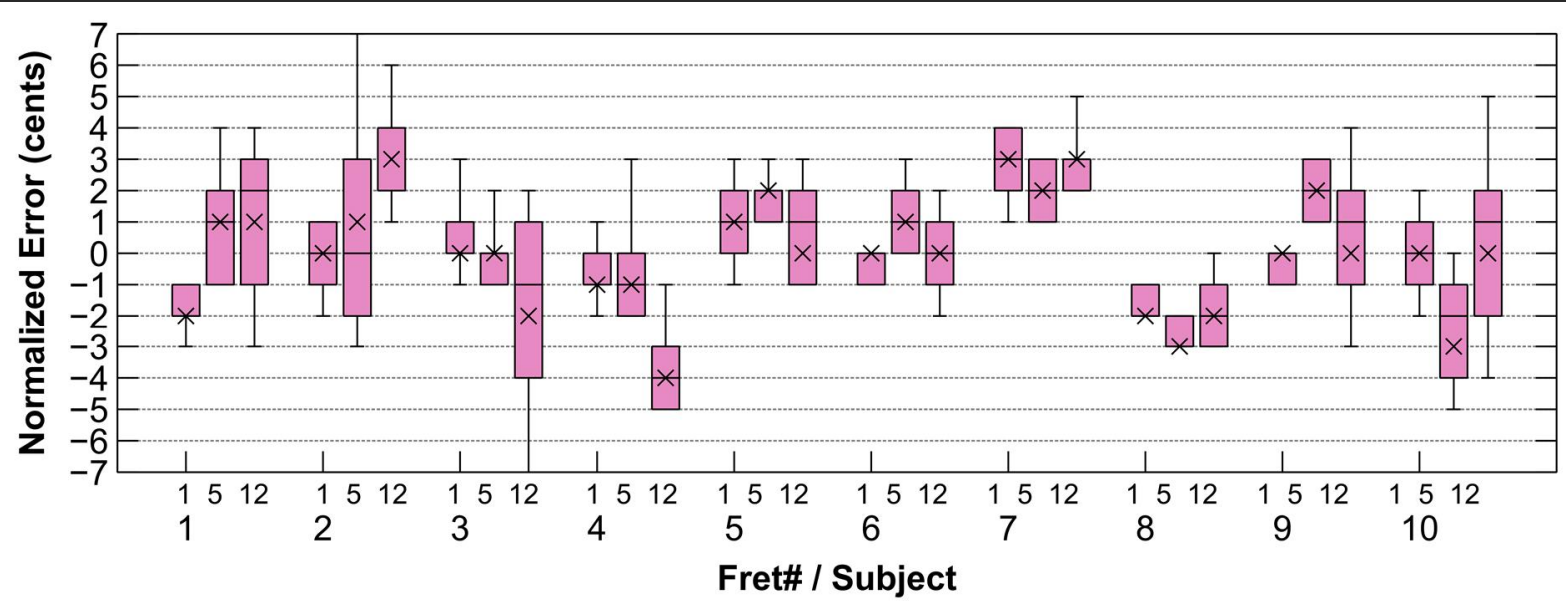

Figure 1 - Pitch variability of three fretted notes, presented grouped by subject and fret as in [1]. Each plot represents 20 same-note samples from 1 subject. Whiskers indicate range minimums and maximums. Box ends span one standard deviation from mean. All data have been normalized so mean value for all study samples of each note (20 samples per subject $\mathrm{x} 10$ subjects $=200)$ is at zero error.

The following observations can be made from this figure:

1. Pitch variability varies among subjects. Some subjects play with more pitch variability than others. Compare subject \#8 with subject \#10 for example;

2. Subjects exhibit individual pitch offset trends independent of variability, i.e. some players tend to play sharper than others. For example, consider the plots for subject \#7 which are entirely above the graph mean, to those of subject \#8 which are entirely below the graph mean;

3. Pitch variability is roughly higher for higher frets, across subjects. Considering just the boxes in the figure, there is rough but noticeable tendency for greater variability of errors for fret \#5 than for fret \#1, and greater variability for fret \#12 than for \#5;

It is possible to derive quantitative measures of each of the above for use in refining guitar intonation models.

An enabling assumption to this analysis is that all measured pitch errors are positive offsets from some nominal pitch. Another way to put this is that all measurable pitch errors are sharp. The assumption is supported by general observations of playing phenomena that can affect pitch. Two that support the assertion are the pitch sharpening effects of increased fretting pressure and of string bending.

It is well understood that a string must be displaced toward the fretboard by some minimum amount beyond the level of the crown of the fret so that the string comes in firm contact with the fret, and thus its vibrating length is stopped by the fret. That minimal string displacement at a given fret defines the lowest pitch that can be achieved at that fret. Additional displacement of the string toward the fretboard will further stretch the string and raise its pitch. At some displacement the string contacts the fretboard surface and so can be displaced no closer to the fretboard, and the resulting pitch is the highest that can be achieved at that fret by displacement toward the fretboard alone.

String bending is another recognized phenomenon[9]. Pitch increases with increased lateral displacement of the point at which the string is fretted. Although this is a recognized playing technique when performed intentionally it is certainly possible to unintentionally laterally deflect the string to some extent during playing, which would also increase pitch some amount. Note that the amount pitch can be raised at a fret by string bending is far greater than the amount it can be raised by fretting displacement described above.

For completeness it should be pointed out that there are two playing phenomena that can lower pitch. These are bending of the neck (which could also increase pitch, depending on whether the neck is bent forward or backward) and "pulling" the fretted string flat. Although neck bending has the potential to change pitch in either direction, it is clear from an analysis of the peripheral open string tuning assessments in [1] that this was not an issue during data collection. Open string pitch remained stable through repeated recordings as the instrument was handled in typical playing fashion. It is unlikely that any "pulling" flat was involved in this experiment either. This playing technique relies 
on friction between finger and string and involves fretting a note and then dragging the fretting finger to stretch the string away from the nut and toward the bridge, thus reducing tension of the vibrating length of the string. The technique works well for classical guitars with their more elastic nylon strings but is not that effective for steel string instruments. In addition, instructions to player subjects in [1] precluded use of such techniques.

Each data point collected in [1] represents a pitch error in whole cents. Each of these data points is the sum of potential component errors, contributed by a number of factors. These include measurement error in the tuner, open string tuning error at the time of data acquisition, intonation error associated with the construction of the guitar, and player induced intonation error. As indicated by the observations of figure 1 above, it may be possible to further divide the latter into a general pitch offset tendency of the player, and separately, pitch error associated with pitch variability. Each of these will be described in detail in the following sections. Where appropriate, methods used to control for or otherwise factor out each component error will be described.

\section{A. Tuner measurement error}

No tuner measurement error was identified in the tuner software used in [1]. To test tuner accuracy, digital audio tone files were generated at note frequency and also at one cent higher and one cent lower. The tuning software accurately reported pitch in all cases.

\section{B. Open string tuning error}

Each data acquisition trial in [1] consisted of 20 notes played by one subject on one string and fret. Prior to the start of the trial the subject played a note on the open string. This playing of the open string note was repeated at the end of the trial as well. Tuning errors of the open string pre and post-trial were compared to determine if there was any tuning degradation during the trial. No tuning degradation was identified for any trial in the experiment. The open string tuning error for each trial was used to correct all 20 fretted note pitch errors for the trial. Thus open string tuning errors were removed prior to subsequent data transformation and analysis. Figure 2 shows pitch error min-tomax ranges for all trials following per-trial correction of open string tuning error present during the trial.

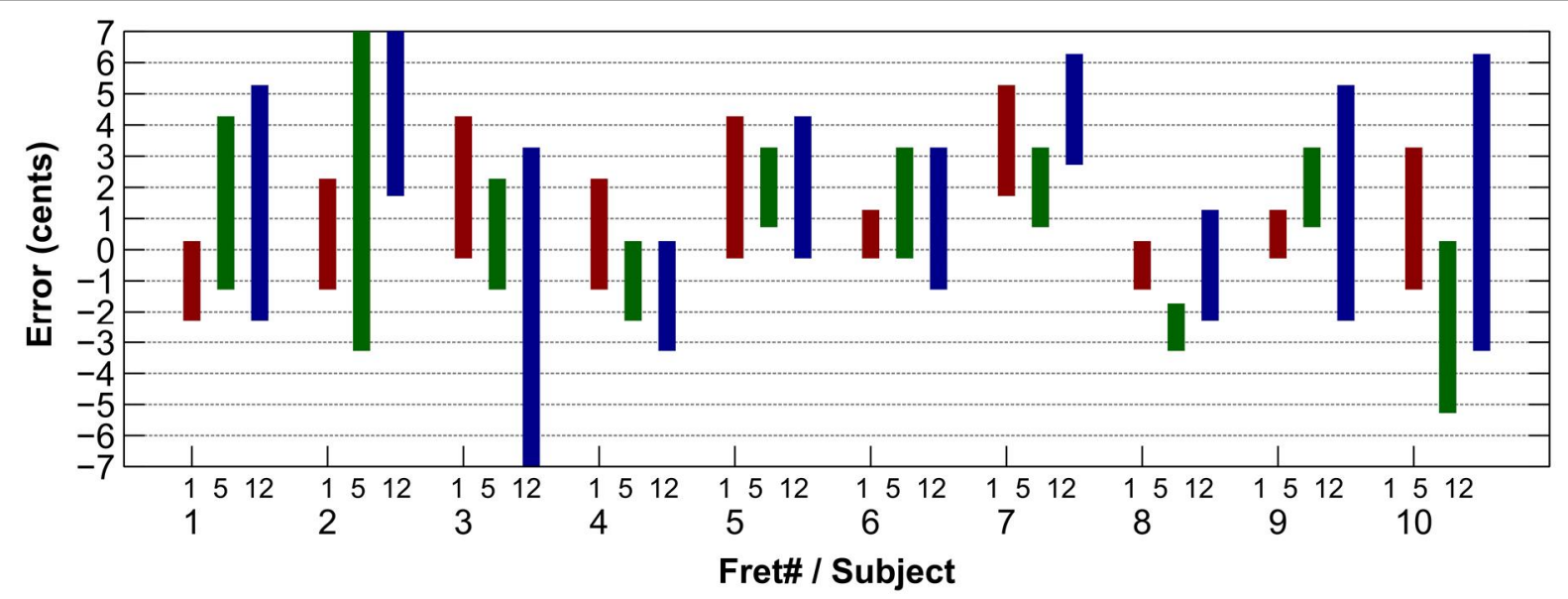

Figure 2 - Ranges of total intonation errors collected in trials in [1] are displayed as vertical bars. These ranges have been corrected for tuning errors of the open string on a per-trial basis. Each error datum in each of the ranges displayed has component parts attributable to the test instrument and to the player. Because players don't introduce pitch flatting errors, the lowest pitch value attained in the study for each fret represents the pitch error for that fret attributable to construction of the instrument.

\section{Error attributable to construction of the guitar}

Construction details of the guitar could result in intonation error at the frets tested. Nut, bridge saddle and fret placement, fret crown placement, neck relief, string height above the fretboard and body resonances can all affect intonation. Included in this list of possible sources of pitch error is the minimum amount of string stretching required to adequately stop the string at the fret. Note that these are all static errors of an instrument and fret, which will be present each time the instrument is fretted. Error from this source was controlled in [1] because all trials were recorded using the same instrument, and so all data points were equally affected by any intonation errors inherent in the construction of that instrument. The error component attributable to construction of the instrument for each fret is represented by the lowest value for that fret achieved by any player in the experiment. This assumes that the lowest 
intonation error achieved by any player in the experiment for a fret position is the lowest possible achievable error at that fret. As can be seen in figure 2, the lowest value achieved for the first fret is -2 cents; for the fifth fret it is -5 cents; and for the twelfth fret it is -7 cents.

For analytical purposes here it is useful to remove this error component from data under consideration so that errors attributable solely to the player can be identified. In figure 3 the lowest error value attained in the experiment for each fret was subtracted from the rest of the error values for that fret. The ranges shown in the figure therefore indicate intonation error that is attributable only to the players.

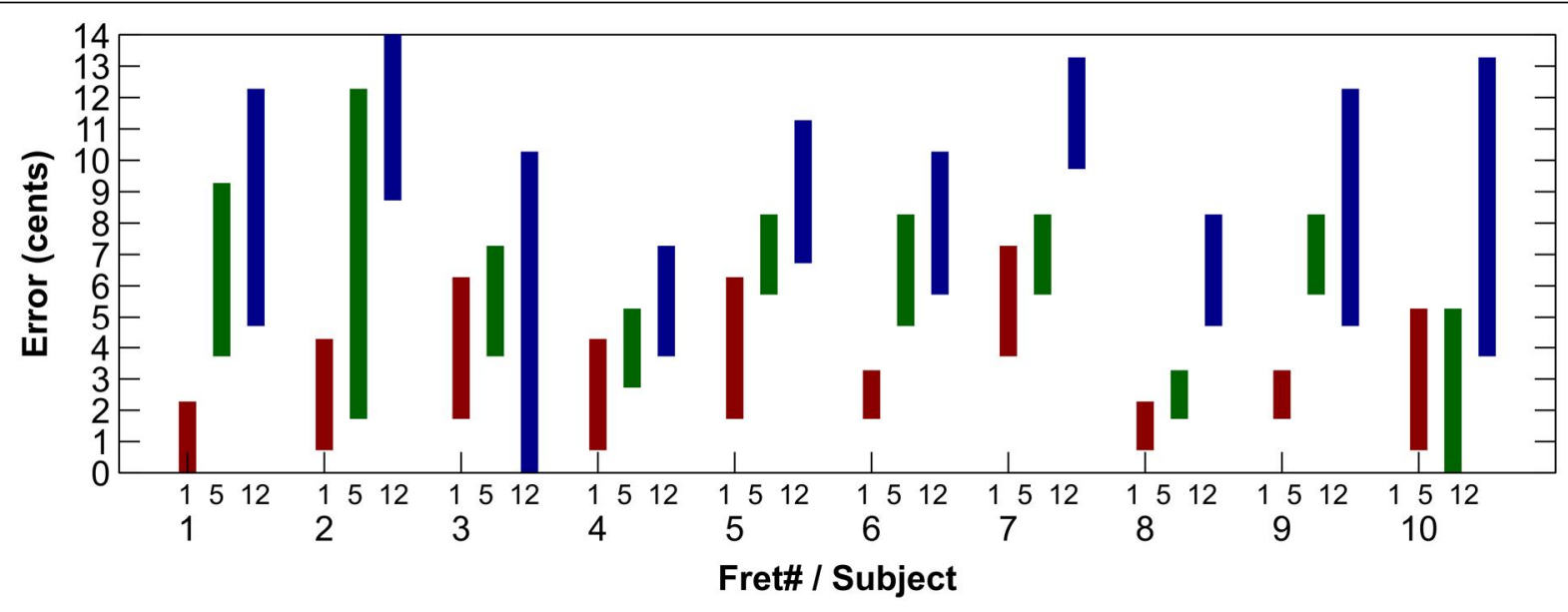

Figure 3 - Ranges of total intonation errors for each fret have been offset by the lowest error value attained for that fret in the experiment. Assuming these base error values are the lowest possible attainable by any player, these values can be assumed to be attributable to the construction of the guitar. This transformation removes error attributable to the construction of the guitar, leaving only error attributable to the players.

\section{Player pitch offset}

It is desirable if a measure of an individual player's pitch offset tendency can be extracted such that it is independent of the individual's pitch variability tendency. Given the data set as currently transformed and assuming the lowest error achieved by a player for a fret position is the lowest level achievable by that player for that fret, these values can serve as indicators of general pitch offset for each player at each of the tested frets. The per-player pitch offset values ranged from $0-4$ cents on fret \#1, $0-6$ cents on fret \#5, and $0-10$ cents on fret \#12. Comparing the offset values for each subject across frets shows a rough tendency for pitch offset values to increase with fret. Despite considerable variability in the offsets across frets for some players, calculated averages of the offsets across frets for each player yield a single per-player average offset value whichmay be useful to indicate a player's general tendency to sharpen the pitch of fretted notes. The variability can be assessed graphically by comparing the individual offset values to their per-player means in figure 4. The lowest average of offset values across frets for a subject was 2 cents, and the highest was 7 cents.

Because the per-player averages across frets were so unrepresentative of central tendency per fret, the component offset values were additionally analyzed with curve fitting software (CurveExpert Professional v1.6). Results are not shown, but indicate that all of these are well fit by relatively simple polynomials. This leaves the problem of deriving per-player coefficient values from available per-player data, a problem which was not addressed in this study.

\section{E. Player pitch variability}

It is desirable if a measure of a player's pitch variability tendency can be extracted such that it is independent of the player's pitch offset tendency. Given the data set as currently transformed, if the entire range of errors for a subject at a fret position is offset so the lowest value of the range is equal to zero, this eliminates the effect of any pitch offset tendency for that subject. The mean value of the resulting range can now be used as an indication of general pitch variability for the subject at that fret, and that mean value can be directly used as a compensation value as well. See figure 5. Per player pitch variability mean values ranged from $0-2$ cents on fret $\# 1,1-4$ cents on fret \#5, and $1-7$ cents on fret \#12. An average of these mean values across frets gives a general indication of pitch variability for a subject. The lowest average of mean errors across frets for a subject was 1 cent, and the highest average was 3 
cents. The general trend, observed in figure 1, of pitch variability increasing with fret number can be seen in figure 5 as well, but the effect is small.

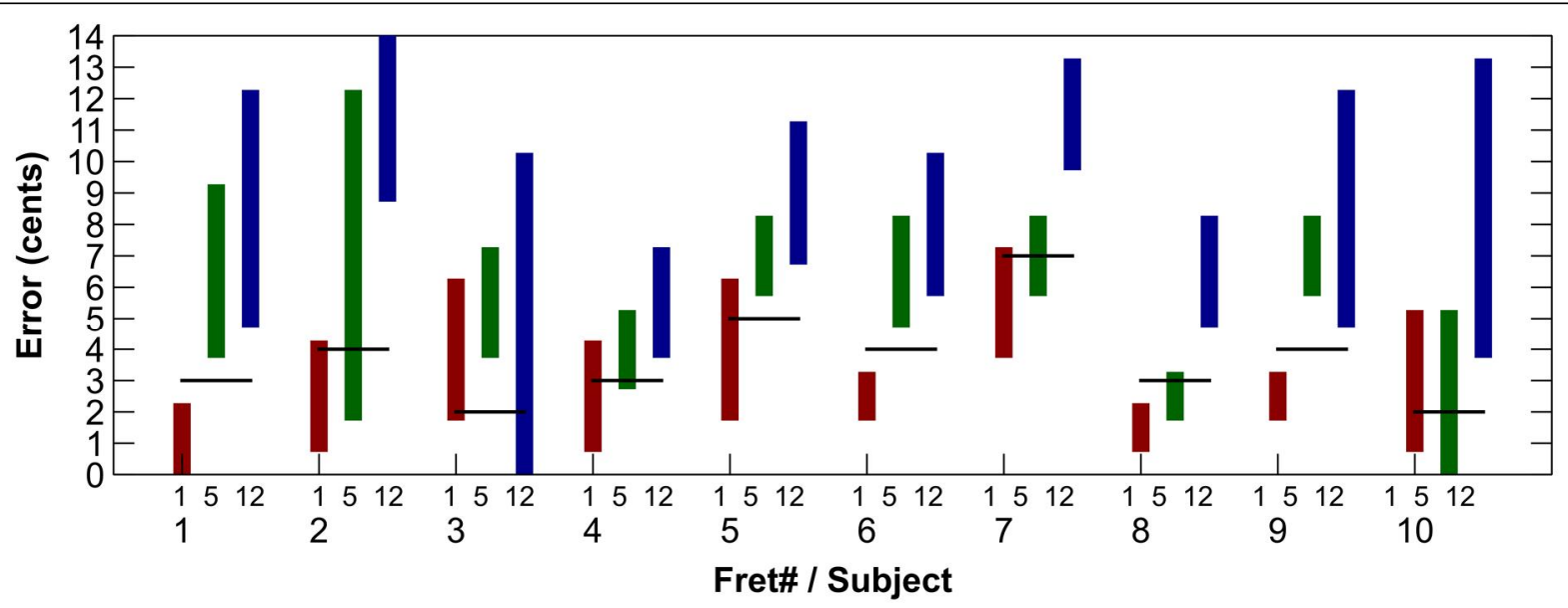

Figure 4 - The lowest value in each range bar indicates the player pitch offset for that player and fret. The black lines show averages of error offset values for all three frets for each player. Due to high variability of the error offset values these averages provide only the roughest approximation of per-player intonation error offsets.

As was the case with player pitch offsets, the per-player pitch variability averages across frets were unrepresentative of central tendency for individual frets. Here too the component offset values were additionally analyzed with curve fitting software with similar results. These are well fit by relatively simple polynomials, but the problem of deriving per-player coefficient values from available per-player data was not addressed in this study.

It was mentioned above that the player pitch variability values can be directly used as compensation values. It is assumed that the extent of same note pitch error induced by the player cannot be eliminated. To compensate for these errors the best that can be done is to subtract a compensation value which will normalize and distribute the error range about zero. The effect of doing so would be to reduce the sharp errors by half but of course to introduce corresponding and currently nonexistent flat errors.

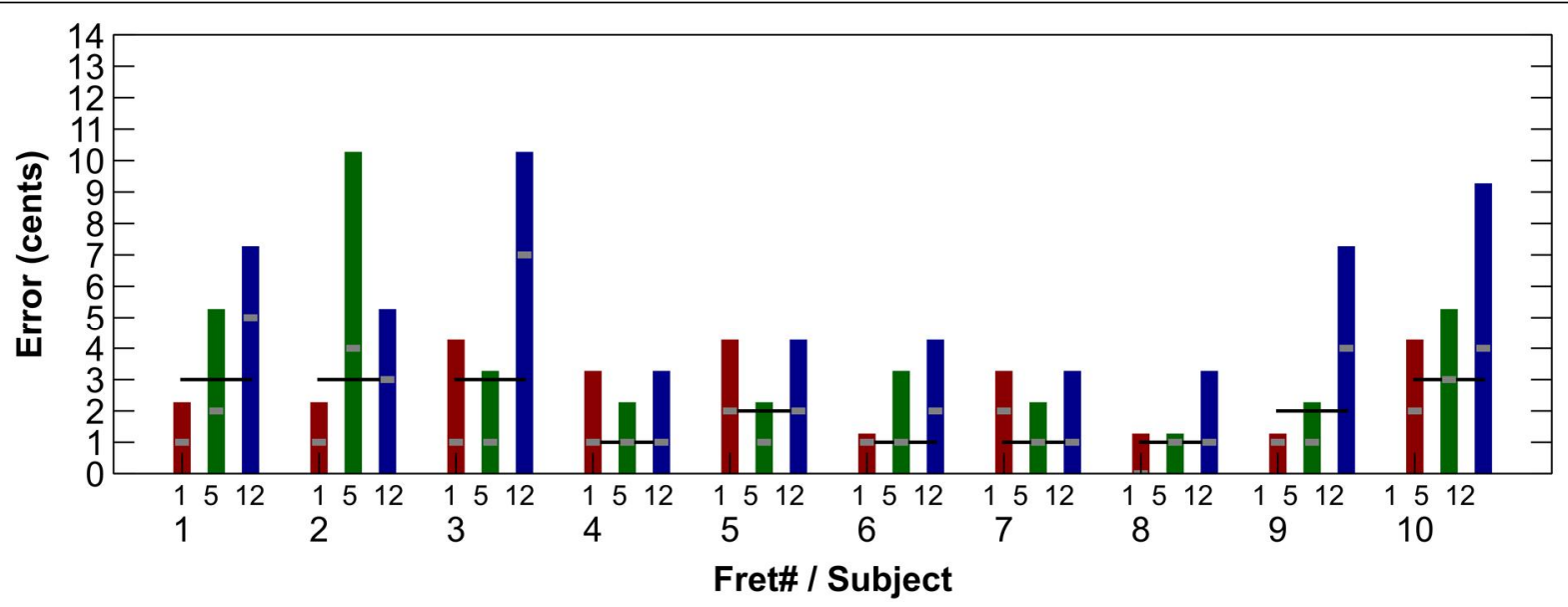

Figure $\mathbf{5}$ - Each range bar appearing infigure 4 has been offset to zero in this figure, thus eliminating player pitch offset tendency. Here, the range bars indicate player pitch variability tendency. The gray bars show means of error variability values for each fret for each player. Average values across frets per player are indicated by the black lines. Due to high variability of the per-fret error values, these averages provide only the roughest approximation of per-player intonation error tendency.

\section{DISCUSSION}

Pitch variability of fretted notes confounds any attempt to simply validate mathematical intonation models from the literature. If enough pitch samples are collected, these can be analyzed to determine pitch error components 
attributable to the instrument and those attributable to the player. The experiment collected limited data from a single string of a single instrument, from only three frets, and from only ten players. The arithmetic operations described here make it possible to describe both the pitch errors induced by the instrument, and the player-induced components of fretted note pitch variability. This information can then be used for two practical purposes. Instrument pitch errors can be used to validate and refine whatever intonation model was used in the design and construction of the instrument, assuming data is collected from enough instruments to make it possible to also identify and isolate any mechanical implementation variability. At least theoretically, instrument-specific static intonation data also may be useful for remedial alterations to an instrument to refine best possible intonation.

Measures of player induced pitch variability may be useful to refine both intonation models and to "tune" an instrument to a player's pitch error tendencies. This study identified minimum per-fret player pitch offset and player pitch variability values that were non-zero. Assuming these values represent population baselines, it may be useful to incorporate these (or similarly obtained) values in proposed general intonation models. Adjusting an instrument to an individual player holds some promise for improved intonation. Some of the intonation improvement articles cited included a player-specific model parameter. This is generally some representation of how much perpendicular string displacement is involved in fretting. Note that in this study it is pretty clear from fret-specific differences that a single parameter value such as this used as a simple displacement offset is not sufficient in capturing either player pitch offset or player pitch variability tendencies.

Figures $\mathbf{4}$ and $\mathbf{5}$ included averages of error values across frets for each player. From visual analysis alone it is obvious that these averages only crudely represent central tendency across frets. Future research may want to consider development of more accurate models.

This study demonstrates intonation error components associated with individual players and frets, based on pitch measurement. It does not consider such error from the perspective of human pitch perception. One study of human perception of differences in intonation effected by typical steel string guitar compensation strategies found that all such strategies were perceived as equivalent by the assessor population[10]. Due to limitations in that study it is not clear if that perceptual limitation applied to all individual assessors. The results of that study raise the possibility that per-player pitch error differences seen in this study may be due to differences in per-player pitch perception thresholds. The possibility also exists that some or all of the pitch variability seen in this study may be below the threshold of human detectability in musical contexts.

\section{CONCLUSION}

Pitch varies with each instance of a played fretted note on a steel string guitar, complicating any attempt to validate interventions intended to improve intonation. An experimental playing context can be configured in which all playerinduced pitch errors are sharp. If enough note samples are collected from enough players for a string and fret of an instrument, the lowest pitch error attained can be assumed to indicate pitch error attributed to construction of the instrument for that string and fret. Subtracting out error attributable to the instrument yields error attributable to the player. The lowest pitch error attained by a player for a string and fret can be used as an indication of that player's tendency to play sharp at that string and fret. Subtracting this value from all samples from a player on that string and fret yields a range of intonation variability for that player on that string and fret. The mean of each such range can be used as a compensation value for the player's intonation variability on that string and fret. Player induced intonation variability can't be eliminated but application of this compensation value can center the range about zero error. Pitch ranges vary widely for each player and fret, making it unlikely that a small number of simple parameters could accurately model the observed variability.

\section{ACKNOWLEDGMENTS}

Thanks to David Cohen for review and comment of a draft of this paper. Thanks to the guitarists that took part in [1] and to the New England Luthiers group which provided access to these subjects at one of its regular meetings.

\section{BIBLIOGRAPHY}

1. Mottola, R.M. (2018, January 18). Same-Fretted-Note Intonation Variability of the Steel String Acoustic Guitar. OSFPreprints. Retrieved from https://osf.io/45mtc/ 
2. Bartolini, W. and Bartolini, P. (1982). Experimental Studies of the Acoustics of Classic and Flamenco Guitars. Journal of Guitar Acoustics 6, 74

3. Gilbert, J. and Gilbert, W. (1984). Intonation and Fret Placement. Soundboard 26-27

4. Byers, G. (2006). Classical Guitar Intonation. American Lutherie \#47 p.34

5. Doolin, M. (2007). Intonation in the Real World. American Lutherie \#92 p.26

6. Gore, T. and Gillet, G. (2011) Contemporary Acoustic Guitar Design and Build

7. Magliari, G. and MacRostie, D. (2013). Beyond the Rule of 18: Intonation for the 21st Century. American Lutherie \#116 p.6

8. Varieschi, G. U. and Gower, C. M. (2010). Intonation and compensation of fretted string instruments. American Journal of Physics, 78(1), 47-55.

9. Mottola, R. M. (2014). Guitar Fretboard Camber and Action in the Context of String Bending. Savart Journal, 1(4).

10. Mottola, R.M. (2017, October 21). Blind Listening Evaluation of Steel String Acoustic Guitar Compensation Strategies. OSFPreprints. Retrieved from https://osf.io/bqzf4/ 